\title{
CONTROLLED RELEASE OF CHLORHEXIDINE FROM A MESOPOROUS SILICA-CONTAINING MACROPOROUS TITANIUM DENTAL IMPLANT PREVENTS MICROBIAL BIOFILM FORMATION
}

\author{
K. De Cremer ${ }^{1,2, \S}$, A. Braem ${ }^{3, \S}$, E. Gerits ${ }^{1}$, K. De Brucker ${ }^{1}$, K. Vandamme ${ }^{4}$, J.A. Martens ${ }^{5}$, J. Michiels ${ }^{1}$, J. Vleugels ${ }^{3}$, \\ B.P.A. Cammue ${ }^{1,2}$ and K. Thevissen ${ }^{1, *}$ \\ ${ }^{1}$ Centre of Microbial and Plant Genetics, KU Leuven, Leuven, Belgium \\ ${ }^{2}$ Department of Plant Systems Biology, VIB, Ghent, Belgium \\ ${ }^{3}$ Department of Materials Engineering, KU Leuven, Leuven, Belgium \\ ${ }^{4}$ Biomaterials-BIOMAT, Department of Oral Health Sciences and Prosthetic Dentistry, \\ KU Leuven and University Hospitals Leuven, Leuven, Belgium \\ ${ }^{5}$ Centre of Surface Chemistry and Catalysis, KU Leuven, Leuven, Belgium
}

$\S$ These authors contributed equally

\begin{abstract}
Roughened surfaces are increasingly being used for dental implant applications as the enlarged contact area improves bone cell anchorage, thereby facilitating osseointegration. However, the additional surface area also entails a higher risk for the development of biofilm associated infections, an etiologic factor for many dental ailments, including peri-implantitis. To overcome this problem, we designed a dental implant composed of a porous titaniumsilica $\left(\mathrm{Ti} / \mathrm{SiO}_{2}\right)$ composite material and containing an internal reservoir that can be loaded with antimicrobial compounds. The composite material consists of a sol-gel derived mesoporous $\mathrm{SiO}_{2}$ diffusion barrier integrated in a macroporous Ti load-bearing structure obtained by powder metallurgical processing. The antimicrobial compounds can diffuse through the porous implant walls, thereby reducing microbial biofilm formation on the implant surface. A continuous release of $\mu \mathrm{M}$ concentrations of chlorhexidine through the $\mathrm{Ti} / \mathrm{SiO}_{2}$ composite material was measured, without initial burst effect, over at least 10 days and using a $5 \mathrm{mM}$ chlorhexidine solution in the implant reservoir. Metabolic staining, CFU counting and visualisation by scanning electron microscopy confirmed that Streptococcus mutans biofilm formation on the implant surface was almost completely prevented due to chlorhexidine release (preventive setup). Moreover, we demonstrated efficacy of released chlorhexidine against mature Streptococcus mutans biofilms (curative setup). In conclusion, we provide a proof of concept of the sustained release of chlorhexidine, one of the most widely used oral antiseptics, through the $\mathrm{Ti} / \mathrm{SiO}_{2}$ material thereby preventing and eradicating biofilm formation on the surface of the dental implant. In principle, our flexible design allows for the use of any bioactive compound, as discussed.
\end{abstract}

Keywords: Porous titanium, mesoporous silica, controlled release, chlorhexidine, Streptococcus mutans, dental implant, in-situ drug delivery, peri-implantitis, biofilm.
*Address for correspondence:

Karin Thevissen

Centre of Microbial and Plant Genetics (CMPG) KU Leuven

Kasteelpark Arenberg 20 box 2460

3001 Leuven, Belgium

Telephone number: +3216329688

Fax number: +32 16321966

Email: karin.thevissen@kuleuven.be

\section{Introduction}

The increased use of implanted medical devices in humans (a trend which is likely to continue with aging populations) largely explains the rise in infectious implant complications. Foreign organisms can be introduced into the body during implant installation and the implant surface often forms an ideal substrate for adhesion of microbial cells, the initial phase of biofilm formation (Busscher et al., 2010; Donelli and Vuotto, 2014; Ribeiro et al., 2012). Peri-implant infections can also occur in later phases of implant therapy. In the case of dental implants for example, infections can occur by bacterial contamination (e.g. due to poor oral hygiene or soft tissue complications) or be induced by other factors (e.g. because of remaining of crown fixation cement). A higher prevalence of periimplantitis has been identified for patients with presence or history of periodontal disease and for smokers (Marcantonio et al., 2015).

The emergence of dental implants has introduced such artificial surfaces on which oral bacteria can form biofilms within the oral cavity (Belibasakis et al., 2015). These biofilms can then trigger infection and inflammation of the peri-implant tissue (i.e. peri-implantitis), leading to chronic infections and progressive peri-implant bone loss in a number of oral surgery patients. Moreover, rough implant surfaces, which were introduced in the dental market in order to improve osseointegration (Bencharit et al., 2014; Taniguchi et al., 2015; Yuan et al., 2014), are thought to facilitate biofilm formation as the total surface area is increased and 'protected' areas are provided (Belibasakis et al., 2015; Braem et al., 2014; Subramani et al., 2009). Still, there is limited and contradictory evidence on the impact 
of implant surface characteristics on peri-implantitis. While several studies reported a positive influence of smooth surfaces on peri-implant health (Esposito et al., 2005; Yuan et al., 2014), others failed to find a correlation between the type of implant surface and marginal bone loss (de Freitas et al., 2011; Nicu et al., 2012; do Prado et al., 2013). Nevertheless, oral biofilm formation seems to be a defining factor for the success or the failure of a dental implant (Subramani et al., 2009) and the prevention and/ or elimination of biofilms at the implant surface is the key to tackling peri-implant infections.

In the case of established biofilms, surgical intervention is often required, highlighting the need for new strategies to prevent biofilm formation on the implant surface. One strategy is the development of implant substrates that can resist biofilm formation by microbial pathogens. This can be acquired by physical or chemical material treatments and/or introduction of antimicrobial agents on the material surface. Newly developed antimicrobial surfaces can largely be classified as anti-biofouling, resisting or preventing microbial attachment, and/or bactericidal, killing microbial cells upon contact (Hasan et al., 2013). This is usually achieved by applying surface coatings of either intrinsically anti-adhesive materials (passive coatings) or antimicrobial releasing carrier materials (active coatings) or by modifying the implant surface by altering the surface topography or the surface chemistry (Campoccia et al., 2013; Hasan et al., 2013).

However, these innovative approaches can also entail important drawbacks such as the fragility of such antiinfective surfaces with respect to the mechanical load during dental implant function and the limited effectiveness of release coatings due to the short timeframe following implantation during which therapeutic concentrations of antimicrobials are released (Hickok and Shapiro, 2012). With this in mind, a novel disk-shaped implant material was developed combining the high strength of a macroporous Ti structure and the drug-release functionality of mesoporous $\mathrm{SiO}_{2}$, enabling a continuous diffusion of bioactive compounds from the feed side to the release side (Braem et al., 2015). In the current study, we aim to realise a proof of concept for the application of this $\mathrm{Ti}$ / $\mathrm{SiO}_{2}$ composite material for a novel dental implant design featuring an internal reservoir which can be (re)loaded with antimicrobial compounds. It is hypothesised that diffusion of antimicrobial compounds through the bulk of the implant from the refillable reservoir can establish a stable continuous release without burst effects over a prolonged period of time, effectively preventing biofilm formation during a longer timeframe. Chlorhexidine was selected as antimicrobial agent in this study, as it is one of the most commonly used and best documented antimicrobials in dentistry. It is available in different forms (mouthwash, gel, aerosol, spray and disks) and is considered to be an effective chemical complement to tooth brushing and flossing (Varoni et al., 2012). The antimicrobial effectiveness of the released chlorhexidine was evaluated using the oral bacterial pathogen Streptococcus mutans ( $S$. mutans).

\section{Materials and Methods}

\section{Composite $\mathrm{Ti} / \mathrm{SiO}_{2}$ disk and implant production}

Macroporous Ti disks ( $\varnothing 12.2 \mathrm{~mm}, \mathrm{~h}=1.3 \mathrm{~mm}$ ) were prepared by powder metallurgy. Ti (grade $\mathrm{S}<8 \mu \mathrm{m}$, Rockwood Lithium, Frankfurt, Germany) and $\mathrm{TiH}_{2}$ starting powders (grade VM, Rockwood Lithium, Frankfurt, Germany) were mixed under argon in a 90:10 molar ratio using a multidirectional mixer (Turbula T2C, WAB, Muttenz, Switzerland) for $24 \mathrm{~h}$. After powder compaction by die pressing at $50 \mathrm{kN}$, the disks were stepwise dehydrogenated [heating at $5{ }^{\circ} \mathrm{C} / \mathrm{min}$ to $450{ }^{\circ} \mathrm{C}(1 \mathrm{~h} \mathrm{dwell}$ time $)$, at $2{ }^{\circ} \mathrm{C} / \mathrm{min}$ to $550{ }^{\circ} \mathrm{C}(1 \mathrm{~h}$ dwell time $)$, at $2{ }^{\circ} \mathrm{C} / \mathrm{min}$ to $650{ }^{\circ} \mathrm{C}(1 \mathrm{~h}$ dwell time $)$ ] and sintered at $850^{\circ} \mathrm{C}$ for $5 \mathrm{~min}$ in a high vacuum furnace $\left(10^{-6} \mathrm{mbar}\right)$. For the production of high-strength Ti dental implants, the processing route was slightly modified in order to obtain cylinders of sufficient length. The starting powder mixture was inserted in polymer sleeves ( $\varnothing 4 \mathrm{~mm}$ prepared by hot dipping; Crocell Super SH25, Lambert Products, Waremme, Belgium) and compacted by cold isostatic pressing at 3000 bar. The resulting cylinders were then stepwise dehydrogenated and consolidated as described above. Sintered cylinders were machined to the final shape using a CNC milling device (TM1, HAAS) applying hard-metal cutting tools and drill bits (DIXI polytool, Le Locle, Switzerland) at high speed. After machining, implants were decontaminated by ultrasonic cleaning in ethanol and acetone for $15 \mathrm{~min}$ each and rinsing in distilled water. In order to remove metal debris and restore the original highly interconnective pore structure as required for mass transport through the bulk of the material, acid etching was performed in a $1.5 \mathrm{M}$ HF (40\%, Sigma-Aldrich, Saint Louis, MO, USA) and 4.5 $\mathrm{M} \mathrm{HNO}_{3}$ (65\%, Chem-Lab, Zedelgem, Belgium) aqueous solution for $5 \mathrm{~s}$. After neutralisation with boric acid (Fisher Scientific, Illkirch, France) and extensive rinsing in distilled water, the samples were cleaned using a mixture of ethanol and isopropanol and stored until further use. A representative number of implants was analysed by scanning electron microscopy (SEM; Nova NanoSEM 450, FEI, Zaventem, Belgium) using backscattered imaging and associated energy dispersive X-ray spectroscopy (EDX, EDAX, Tilburg, The Netherlands) to check for organic residue in order to confirm that the samples were sufficiently clean.

Integration of the mesoporous $\mathrm{SiO}_{2}$ diffusion barrier was done by sol-gel synthesis. A mixture (1:1 volume) of a colloidal $\mathrm{SiO}_{2}$ suspension (Ludox ${ }^{\circledR}$ HS-40, SigmaAldrich, Saint Louis, MO, USA) and an aqueous $0.055 \mathrm{M}$ $\mathrm{HCl}$ (37\%, Chem-Lab, Zedelgem, Belgium) solution was magnetically stirred for $10 \mathrm{~min}$. Using an in-house designed sample holder, this sol was injected through the disks or through the internal reservoir of the dental implants using a high pressure pump (880-PU, Jasco, Easton, MD, USA) until the sol could be observed on the outside, to ensure the complete sealing of the internal reservoir from the implant surroundings. Samples were aged overnight at $60{ }^{\circ} \mathrm{C}$ followed by a heat treatment at $225^{\circ} \mathrm{C}$ in air for $6 \mathrm{~h}$ $\left(1{ }^{\circ} \mathrm{C} / \mathrm{min}\right.$ heating rate, RHF 1200 , Carbolite Furnaces, Aartselaar, Belgium) in order to decompose surfactant templates (calcination). 

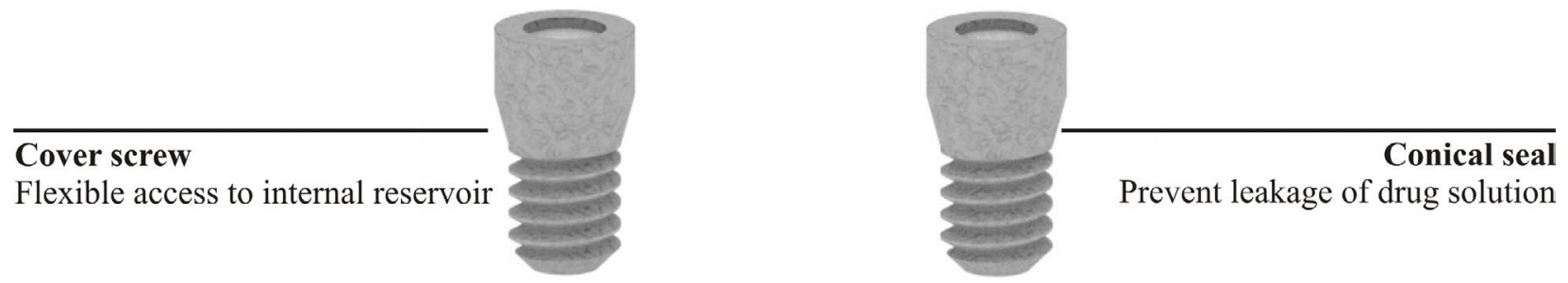

Cover screw

Flexible access to internal reservoir
Prevent leakage of drug solution

External hex connector Implant installation and

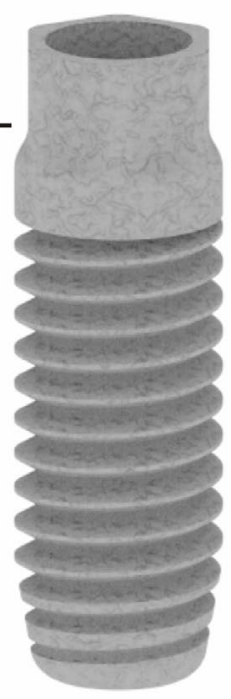

crown seating

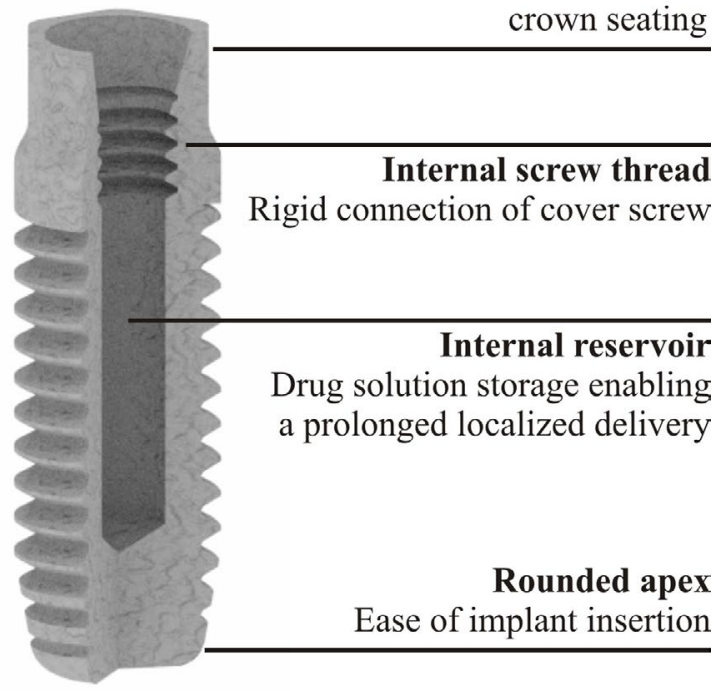

Fig. 1. Conceptual design for a dental implant enabling a sustained drug release. The one-piece implant/abutment configuration is made out of a mesoporous $\mathrm{Ti} / \mathrm{SiO}_{2}$ composite material allowing diffusion of drug molecules from an internal reservoir to the implant surface and surroundings. A cover screw allows easy refilling of the reservoir while the conical seal system prevents leakage of the drug solution to the oral cavity.

\section{Implant design and characterisation}

The design of the dental implants is presented in Fig. 1. It was designed to comprise a one piece implant/abutment configuration (Ø $3.5 \mathrm{~mm}, 8 \mathrm{~mm}$ intraosseous length, $3 \mathrm{~mm}$ abutment length) for one step surgical installation and immediate restoration without the need of a second surgery for abutment connection. Moreover, an internal reservoir ( $\varnothing 1.6 \mathrm{~mm}, 8 \mathrm{~mm}$ of depth) was incorporated in the design for customised drug solution storage allowing prolonged release of antimicrobial compounds through the mesoporous $\mathrm{Ti} / \mathrm{SiO}_{2}$ to the implant surface and surroundings. The intraosseous part consists of a M3.5 double lead thread, while the abutment is featured with an external hex connection (hexagonal diameter $3.2 \mathrm{~mm}$ ). To improve the ease of insertion and to avoid stress concentrations at the implant apex, the apex is slightly rounded $(\mathrm{R} 0.5 \mathrm{~mm})$. A stainless steel cover screw $(\mathrm{M} 2 \times 2.25)$ for flexible refilling of the internal reservoir is equipped with a conical surface mating the $2^{\circ}$ countersink in the implant abutment in order to provide a sealing surface that effectively prevents leakage of the drug solution to the oral cavity and vice versa. A representative number of polished cross-sections of Ti dental implants, before and after incorporation of the mesoporous $\mathrm{SiO}_{2}$ diffusion barrier, were examined by SEM and EDX, in order to confirm that the $\mathrm{SiO}_{2}$ phase was uniformly distributed into the Ti macropores in a consistent way. Pore characteristics of the porous Ti dental implants after machining and acid etching were measured by mercury intrusion porosimetry (MIP, AutoPore IV 9500, Micromeretics, Norcross, GA, USA). Characterisation of the mesoporous $\mathrm{SiO}_{2}$ diffusion barrier was done by nitrogen adsorption/desorption at $-196{ }^{\circ} \mathrm{C}$ (Autosorb 1, Quantachrome, Boynton Beach, FL, USA) using the Brunauer-Emmett-Teller (BET) method and the Density Functional Theory (DFT) method on the adsorption branch of the isotherm to determine the specific surface area and the pore size distribution, respectively. As pre-treatment to remove adsorbed contaminants, the samples were evacuated at $200{ }^{\circ} \mathrm{C}$ for $12 \mathrm{~h}$.

\section{Strain and chemicals for release experiments}

The $S$. mutans strain Clarke, originally isolated from dental caries (Clarke, 1924), was grown routinely on tryptic soy broth (TSB) agar plates (Becton Dickinson Benelux, Erembodegem, Belgium) at $37^{\circ} \mathrm{C}$ for $2 \mathrm{~d}$. Overnight cultures were grown in liquid TSB medium.

Stock solutions of chlorhexidine diacetate salt hydrate (Sigma-Aldrich, Saint Louis, MO, USA) were prepared in Milli-Q water (Merck Millipore, Overijse, Belgium). Artificial gingival crevicular fluid (GCF medium) comprised $40 \%$ human serum and $60 \%$ RPMI medium [RPMI 1640 (pH 7.0) with L-glutamine and without 


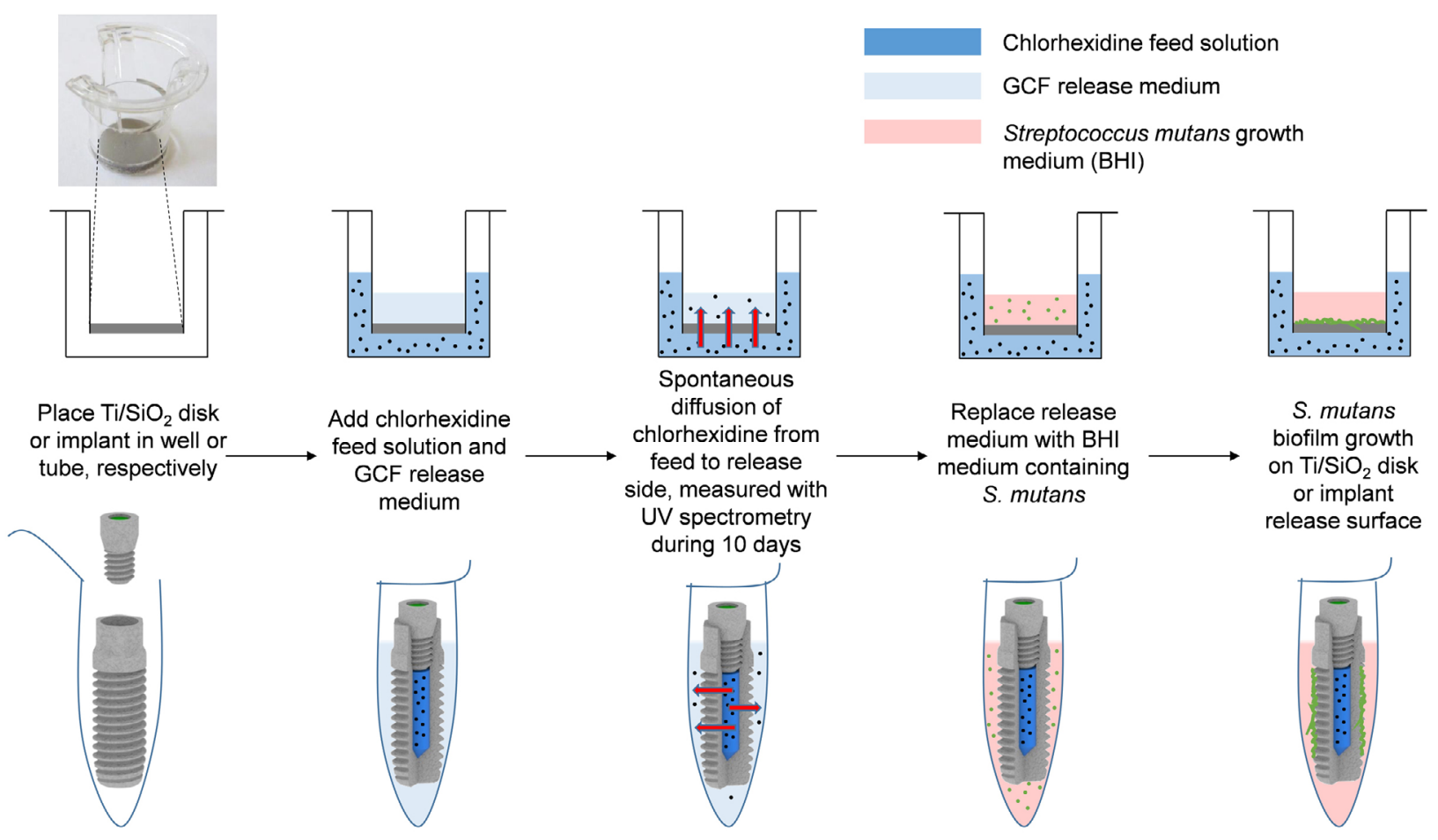

Fig. 2. Schematic representation of the experimental setup of chlorhexidine release experiments and biofilm prevention using $\mathrm{Ti} / \mathrm{SiO}_{2}$ disks or implants. The $\mathrm{Ti} / \mathrm{SiO}_{2}$ material [grey disk or implant (upper and lower part, respectively)] is placed in its respective container. Chlorhexidine or control solutions (dark blue) and GCF medium (light blue) are administered to feed and release compartments of both settings, respectively. The spontaneous diffusion of chlorhexidine from feed to release side is measured each $2 \mathrm{~d}$ using UV spectrophotometry. After $10 \mathrm{~d}$, the GCF release medium is replaced with BHI medium (pink) containing approximately $2 \times 10^{6} \mathrm{~S}$. mutans cells $/ \mathrm{mL}$ (green dots) to allow bacterial biofilm formation on the implant material (biofilm prevention). After $72 \mathrm{~h}$, biofilm formation is quantified using metabolic staining and CFU counting or visualised by SEM imaging.

sodium bicarbonate buffered with MOPS (all SigmaAldrich, Saint Louis, MO, USA)]. The GCF medium was supplemented with $0.5 \mu \mathrm{g} / \mathrm{mL}$ menadione and $5.0 \mu \mathrm{g} /$ $\mathrm{mL}$ hemin (both Sigma-Aldrich, Saint Louis, MO, USA) as described previously (Dalwai et al., 2006). The BHI (brain-heart infusion) medium (Becton Dickinson Benelux, Erembodegem, Belgium) was supplemented with $3 \%$ sucrose (VWR International, Radnor, PA, USA) for $S$. mutans biofilm growth (Junka et al., 2015). Phosphate buffered saline (PBS) comprised $8 \mathrm{~g} / \mathrm{L} \mathrm{NaCl}, 0.2 \mathrm{~g} / \mathrm{L} \mathrm{KCl}$, $1.44 \mathrm{~g} / \mathrm{L} \mathrm{Na}_{2} \mathrm{HPO}_{4}$ and $0.24 \mathrm{~g} / \mathrm{L} \mathrm{KH}_{2} \mathrm{PO}_{4}(\mathrm{pH} 7.4$; all VWR International, Radnor, PA, USA).

\section{Chlorhexidine $\mathrm{Ti} / \mathrm{SiO}_{2}$ disk release experiments}

Release experiments using chlorhexidine and the $\mathrm{Ti} / \mathrm{SiO}_{2}$ composite material shaped in disks were performed in our previously developed in vitro test tool (Braem et al., $2015)$ with slight alterations which are described here (Fig. 2). Solutions based on Milli-Q water containing different concentrations of chlorhexidine $(0,0.1,0.25$ and $1 \mathrm{mM}$ ) (termed feed), were supplied to 12-well plates (1.4 mL/well, 5 wells/condition). $\mathrm{Ti} / \mathrm{SiO}_{2}$ disks sealed into polystyrene cups were decontaminated with $100 \%$ ethanol (VWR International, Radnor, PA, USA) and dried under sterile conditions. Sterile disks were placed in the wells of the 12-well plate, thereby assuring contact between disk and feed solution. On the release side of the $\mathrm{Ti} / \mathrm{SiO}_{2}$ disk, we administered $300 \mu \mathrm{L}$ GCF medium, mimicking natural gingival crevicular fluid (Dalwai et al., 2006), a fluid that is present in the tight anatomical cavity (the socalled periodontal pocket) formed between tooth or implant and periodontal mucosa. Next, the plates were placed in a humidified box to avoid evaporation. The GCF release medium was replaced each $2 \mathrm{~d}$ to maintain sink conditions. Chlorhexidine release at $37{ }^{\circ} \mathrm{C}$ from the feed side to the release side of the $\mathrm{Ti} / \mathrm{SiO}_{2}$ disk was measured in $100 \mu \mathrm{L}$ of the release medium by UV spectrophotometry [in UV Star microplates (Greiner Bio-One GmbH, Frickenhausen, Germany) with a Synergy Mx multi-mode microplate reader (Biotek, Winooski, VT, USA)]. Absorbance levels were corrected for background absorbance by subtracting the absorbance level of GCF medium recovered from control $\mathrm{Ti} / \mathrm{SiO}_{2}$ disks (blank feed solution). A series of solutions containing 6.25-200 $\mu \mathrm{M}$ chlorhexidine in GCF medium were prepared fresh for each day of measurement, in the original GCF medium that was used for the release experiment, to develop a calibration curve. The UV absorbance of $100 \mu \mathrm{L}$ of each suspension was measured, confirming a linear relationship between absorbance at $255 \mathrm{~nm}$ and chlorhexidine concentration over the entire 
concentration range that was tested $\left(\mathrm{R}^{2}=0.96-0.98\right)$. We performed two experiments using two or three independent $\mathrm{Ti} / \mathrm{SiO}_{2}$ disks in each experiment $(n=5)$.

To get an indication of the diffusion regime, assuming that the release of chlorhexidine is governed by Fickian diffusion, an estimate of the order of magnitude of the apparent diffusivity was calculated. Fick's first law was applied based on the obtained release data and dimensions of the release system (note that the macropores which contain the $\mathrm{SiO}_{2}$ phase only take up $30 \%$ of the total surface area of the disks) and assuming sink conditions (since the release medium was regularly refreshed) and a path length equal to the thickness of the disks. The value presented is the mean $\pm \mathrm{SD}$.

\section{$\mathrm{Ti} / \mathrm{SiO}_{2}$ disk biofilm prevention experiment}

After $10 \mathrm{~d}$ of chlorhexidine release through the $\mathrm{Ti} / \mathrm{SiO}_{2}$ disks, the GCF release medium was replaced by $300 \mu \mathrm{L}$ BHI medium containing approximately $2 \times 10^{6} \mathrm{~S}$. mutans cells $/ \mathrm{mL}$ (Fig. 2). Biofilms of $S$. mutans were allowed to grow on the release side of the $\mathrm{Ti} / \mathrm{SiO}_{2}$ disks for $72 \mathrm{~h}$ at $37^{\circ} \mathrm{C}$ (renewal of BHI after $48 \mathrm{~h}$ ), washed with PBS and quantified with the viability dye CellTiter-Blue ${ }^{\circledR}$ (CTB, Promega, Madison, WI, USA) by adding $300 \mu \mathrm{L}$ CTB diluted $1 / 10$ in PBS to each well. After $2 \mathrm{~h}$ of incubation in the dark at $37{ }^{\circ} \mathrm{C}$, fluorescence was measured with a fluorescence spectrometer (Synergy Mx multi-mode microplate reader (Biotek, Winooski, VT, USA) at an excitation wavelength of $535 \mathrm{~nm}$ and an emission wavelength of $590 \mathrm{~nm}$. Fluorescence values of the samples were corrected by subtracting the average fluorescence value of CTB of uninoculated disks (blank). Percentage of metabolically active biofilm cells was calculated relative to the control treatment (blank feed solution). We performed two experiments using two or three independent $\mathrm{Ti} / \mathrm{SiO}_{2}$ disks in each experiment $(n=5)$. Results were analysed for statistical significance by one-way ANOVA and Bonferroni's correction for multiple testing.

S. mutans biofilms grown on control $\mathrm{Ti} / \mathrm{SiO}_{2}$ disks and on $\mathrm{Ti} / \mathrm{SiO}_{2}$ disks placed in $1 \mathrm{mM}$ chlorhexidine feed solution were visualised by SEM. Prior to SEM analysis, samples were carefully washed in PBS to remove nonadherent cells and fixed with glutaraldehyde [2.5\% in a cacodylate buffer (0.1 M, pH 7.4); both Sigma-Aldrich, Saint Louis, MO, USA] for $30 \mathrm{~min}$ and rinsed 3 times with PBS, followed by dehydration in a series of ethanol washes (30, 50, 70, 90 and $100 \%$ ethanol for 20 min each). Afterwards samples were dried and coated with an Au-Pd layer using a sputtering device (Edwards S150) in order to produce a thin conductive film on the surface. SEM was operated at standard high-vacuum settings at $5 \mathrm{~mm}$ working distance and $10 \mathrm{keV}$ accelerating voltage. For samples containing large amounts of organic (biofilm) material at the surface, low-energy imaging using beam deceleration was performed by applying a stage (and sample) bias field, resulting in an effective landing energy of $5 \mathrm{keV}$.

\section{$\mathrm{Ti} / \mathrm{SiO}_{2}$ disk curative biofilm experiment}

Biofilms of $S$. mutans were allowed to grow on the release side of the $\mathrm{Ti} / \mathrm{SiO}_{2}$ disks by adding $300 \mu \mathrm{L} \mathrm{BHI}$ medium containing approximately $2 \times 10^{6} \mathrm{~S}$. mutans cells $/ \mathrm{mL}$.
After incubation for $72 \mathrm{~h}$ at $37^{\circ} \mathrm{C}$ (renewal of BHI after $48 \mathrm{~h}), 1.4 \mathrm{~mL}$ of chlorhexidine $(0.25 \mathrm{mM})$ or blank control solution was added to the feed compartment. Two days later, biofilms were washed with PBS and quantified with CTB, as described above. Moreover, the amount of colony forming units (CFUs) was determined in biofilms grown on control and chlorhexidine-releasing implants. To this end, the disks were washed with PBS, vortexed vigorously for $1 \mathrm{~min}$, sonicated for $10 \mathrm{~min}$ at $45,000 \mathrm{~Hz}(71.4 \mathrm{~W} / \mathrm{L})$ in a water bath sonicator (VWR USC 300-T) and vortexed again. The detached cells were pelleted by centrifugation and washed once more with PBS. A dilution series was established in PBS and $100 \mu \mathrm{L}$ of each dilution was plated onto TSA plates. After incubation at $37^{\circ} \mathrm{C}$ for at least $24 \mathrm{~h}$, the number of CFUs was counted for each condition. The experiment was performed using three independent $\mathrm{Ti} / \mathrm{SiO}_{2}$ disks $(n=3)$. Values are presented as mean \pm SEM. Results were analysed for statistical significance by unpaired student's $t$-test.

\section{Chlorhexidine $\mathrm{Ti} / \mathrm{SiO}_{2}$ implant release experiments and biofilm prevention}

Release experiments using chlorhexidine and the Ti/ $\mathrm{SiO}_{2}$ composite material shaped in our novel implant design were performed in analogy to the $\mathrm{Ti} / \mathrm{SiO}_{2}$ disk experiments (Fig. 2). $\mathrm{Ti} / \mathrm{SiO}_{2}$ implants and cover screws were decontaminated with $100 \%$ ethanol and dried under sterile conditions. A solution (termed feed) containing chlorhexidine $(5 \mathrm{mM})$ or blank control solution was administered to the internal reservoir of the implant (10 $\mu \mathrm{L} /$ implant, 3 implants/condition) before closing the reservoir with the cover screw. The implant was placed in a $0.5 \mathrm{~mL}$ Eppendorf tube which contained $90 \mu \mathrm{L}$ of the GCF medium (termed release) and the tube was closed. The GCF release medium was replaced after each $2 \mathrm{~d}$ to maintain sink conditions. Chlorhexidine release at $37^{\circ} \mathrm{C}$ from the internal reservoir of the implant (feed side) to the release side of the $\mathrm{Ti} / \mathrm{SiO}_{2}$ implant (containing the $\mathrm{GCF}$ medium) was measured in $70 \mu \mathrm{L}$ of the release medium by UV spectrophotometry as described above. We performed two experiments using three independent $\mathrm{Ti} / \mathrm{SiO}_{2}$ disks in each experiment $(n=6)$.

After $10 \mathrm{~d}$ of chlorhexidine release through the $\mathrm{Ti} / \mathrm{SiO}_{2}$ implants, the release medium was replaced by $90 \mu \mathrm{L}$ BHI medium containing approximately $2 \times 10^{6} \mathrm{~S}$. mutans cells/ $\mathrm{mL}$ (6 implants/condition). Biofilms of $S$. mutans were allowed to grow in the same conditions as described above on the release side of the $\mathrm{Ti} / \mathrm{SiO}_{2}$ implants. Quantification of biofilm metabolic activity was performed as described above using $200 \mu \mathrm{L}$ diluted CTB. Moreover, the amount of CFUs was determined as described above.

To assure that our sonication procedure had no effect on the viability of the $S$. mutans cells, $1 \mathrm{~mL}$ of a 10 times diluted overnight $S$. mutans culture was subjected to the sonication procedure. A CFU plating experiment confirmed no effect of sonication on cell viability $(p$-value $=0.859$ in unpaired two-tailed students $t$-test $; n=3$ ). Control (without sonication) and sonicated cell cultures both contained approximately $2 \times 10^{7} \mathrm{CFUs}$. This cell number was chosen to represent the number of CFUs present in control biofilms formed on the implant surface. 


\section{Minimum (biofilm) inhibitory concentration}

Minimum inhibitory concentration (MIC) assays were performed in liquid TSB medium. Briefly, overnight cultures of bacteria were diluted in fresh medium to a final concentration of approximately $5 \times 10^{5}$ cells $/ \mathrm{mL}$. Next, bacteria were incubated in the presence of a twofold dilution series of chlorhexidine. After $24 \mathrm{~h}$ of growth at $37^{\circ} \mathrm{C}$, bacterial growth was determined by measuring the $\mathrm{OD}_{595^{\circ}}$ MIC was interpreted as the lowest antibiotic concentration that completely inhibits bacterial growth.

To assess the anti-biofilm activity of chlorhexidine against $S$. mutans, overnight cultures were diluted $1 / 200$ in BHI medium and two-fold serial dilutions $(150 \mu \mathrm{L})$ of chlorhexidine in the cell suspensions were prepared in a microtiter plate. After $24 \mathrm{~h}$ of biofilm formation at $37^{\circ} \mathrm{C}$, biofilms were washed with PBS and quantified with CTB as described above using $200 \mu \mathrm{L}$ diluted CTB. The lowest concentration of chlorhexidine required to inhibit biofilm formation was defined as the biofilm inhibitory concentration (BIC).

\section{Cytotoxicity assay}

A cytotoxicity test of chlorhexidine was performed on a cell type relevant to bone homeostasis. MG63 osteoblastlike cells, a human osteosarcoma cell line, were obtained from ATCC (American Type Culture Collection CRL1427, LGC Standards, Molsheim, France). Cells were plated in 24-well plates at 2000 cells $/ \mathrm{cm}^{2}$ in Minimum Essential Medium Eagle Alpha Modification ( $\alpha$ MEM; Sigma-Aldrich, Saint Louis, MO, USA) with 0.292 g/L L-glutamine (G7513, Sigma-Aldrich, Saint Louis, MO, USA) supplemented with $10 \%$ foetal bovine serum (PAA Laboratories $\mathrm{GmbH}$, Pasching, Austria) and $1 \%$ antibioticantimycotic (Gibco 15240, Life Technologies SAS, Saint Aubin, France). Cells were maintained overnight at $37^{\circ} \mathrm{C}$ in a humidified environment with $5 \% \mathrm{CO}_{2}$. The media were changed every $48 \mathrm{~h}$.

At day 2 post seeding, cells were incubated with chlorhexidine by adding the compound to the culture medium. As a control, a suspension of the same cell line under the same conditions, but without chemicals, was cultured. Dilution assays started at the maximum concentration of $10 \mu \mathrm{M}$. Chlorhexidine concentrations were freshly prepared at each time point of addition. The proliferation of the MG63 cells in the presence or absence of chlorhexidine was live investigated by means of a resazurin-based reagent (Presto Blue $\AA$, Thermo Fischer, Breda, The Netherlands) after 2, 4 and $6 \mathrm{~d}$ of compound addition. The Presto Blue solution (PB) (Life Technologies, Ghent, Belgium) was prepared in a proportion of 10 unit volumes of medium and 1 unit of reagent. The medium in each well was removed and replaced by $0.5 \mathrm{~mL}$ of PB. After $2 \mathrm{~h}$ incubation, 2 aliquots of $200 \mu \mathrm{L}$ were taken from each well and transferred to a 96-well plate. The PB solution was completely removed from each well and replaced by $1 \mathrm{~mL}$ of culture medium containing the appropriate chlorhexidine concentration. The fluorescent signal was measured with an automated microplate fluorimeter (SerColab Systems, Merksem, Belgium). The blank value was subtracted from the measurement of each group analysed. The experiment was performed twice with duplicate measurements.

\section{Results}

Chlorhexidine is released in a controlled way through $\mathrm{Ti} / \mathrm{SiO}_{2}$ composite disks and inhibits $S$. mutans biofilm formation

We used our previously developed in vitro test tool (Braem et al., 2015) consisting of the $\mathrm{Ti} / \mathrm{SiO}_{2}$ composite material shaped into disks (Fig. 2, upper part), as a step-up model to assess the release of the antimicrobial compound chlorhexidine through the mesoporous material and to design the novel implant.

The $10 \mathrm{~d}$ release profile of chlorhexidine through the $\mathrm{Ti} / \mathrm{SiO}_{2}$ disks, containing different feed concentrations $(0.1$, 0.25 and $1 \mathrm{mM}$ ), is shown in Fig. 3a. A steady release of chlorhexidine was apparent from day 3 after the start of the experiment (illustrated by the nearly linear cumulative release profile). Feed solutions of $0.1,0.25$ and $1 \mathrm{mM}$ chlorhexidine released on average 9,25 and $99 \mathrm{nmol} / \mathrm{cm}^{2}$, respectively (corresponding to $33 \mu \mathrm{M}, 89 \mu \mathrm{M}$ and $359 \mu \mathrm{M}$ in the release medium). Higher chlorhexidine release was observed for higher feed concentrations at all time points. Moreover, we were able to detect chlorhexidine $(>6.25 \mu \mathrm{M})$ in the release medium up to $40 \mathrm{~d}$ after the start of the experiment (using $1 \mathrm{mM}$ chlorhexidine feed solution). Based on these release data and assuming that the release of chlorhexidine through the $\mathrm{SiO}_{2}$ phase of the $\mathrm{Ti} / \mathrm{SiO}_{2}$ disks is governed by Fickian diffusion, the apparent diffusivity as averaged over the different feed concentrations, was estimated to be $2.0 \pm 0.1 \times 10^{-11} \mathrm{~m}^{2} / \mathrm{s}$.

Next, we assessed whether the chlorhexidine-releasing material could prevent biofilm formation in vitro. Fig. $3 \mathbf{b}$ shows the metabolic activity of $S$. mutans biofilm cells grown on the release side of $\mathrm{Ti} / \mathrm{SiO}_{2}$ disks that were actively releasing chlorhexidine. The metabolic activity of biofilm cells was significantly decreased $( \pm 99 \%$ reduction) for all chlorhexidine feed concentrations as compared to blank feed solutions. Bacterial growth on control $\mathrm{Ti} / \mathrm{SiO}_{2}$ disks and on chlorhexidine-releasing $\mathrm{Ti} / \mathrm{SiO}_{2}$ disks $(1 \mathrm{mM}$ feed solution) was visualised by SEM imaging (Fig. 4). Thick $S$. mutans biofilms were formed only on control $\mathrm{Ti} / \mathrm{SiO}_{2}$ disks releasing no chlorhexidine. Interconnected mushroomshaped micro-colonies, consisting of coccus-shaped $S$. mutans cells, were clearly visible within the biofilm, which was spread out over the entire disk surface, covering all pores. On the contrary, the open porosity of the $\mathrm{Ti} / \mathrm{SiO}_{2}$ material was still apparent in the chlorhexidine-releasing disks, which contain only few attached $S$. mutans cells (Fig. 4). The lowest released chlorhexidine concentration $(33 \mu \mathrm{M})$ was able to kill a $S$. mutans culture in a normal vial of BHI medium in less than $24 \mathrm{~h}$. Inhibition of $S$. mutans by chlorhexidine at the released concentrations was expected, since they are well above the MIC $(3.2 \mu \mathrm{M})$ and BIC $(1.6 \mu \mathrm{M})$ values. It should be noted that the data obtained in the preventive $\mathrm{Ti} / \mathrm{SiO}_{2}$ disk setup were not validated by $\mathrm{CFU}$ counting. In this respect, the disk setup should be considered as a preliminary tool which enables us to (relatively quickly) screen more concentrations and compounds (see also Braem et al., 2015). Therefore, only metabolic staining and SEM imaging were used to quantify and visualise biofilm formation in the preventive setup using $\mathrm{Ti} / \mathrm{SiO}_{2}$ disks. The following experiments (curative 
(a)
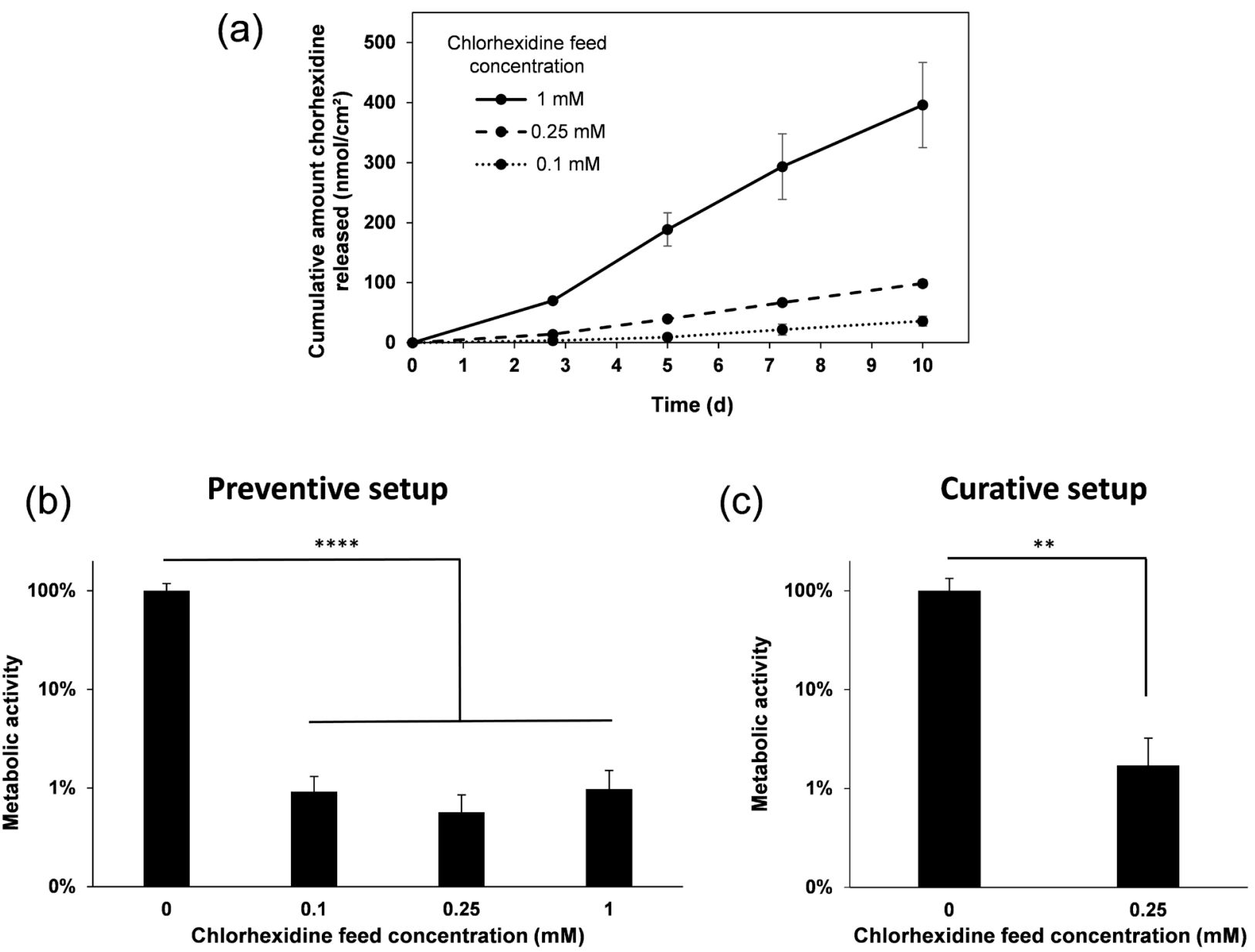

Fig. 3. Release of chlorhexidine through $\mathrm{Ti} / \mathrm{SiO}_{2}$ disks and associated effect on $S$. mutans biofilm metabolic activity. (a) Cumulative amount of chlorhexidine released through $\mathrm{Ti} / \mathrm{SiO}_{2}$ disks in the in vitro test tool for different chlorhexidine feed concentrations (measured by UV spectrophotometry at $255 \mathrm{~nm}$ ). Values are mean \pm standard error of two independent experiments containing 2-3 repeats $(n=5)$. (b) Prevention of $S$. mutans biofilms on $\mathrm{Ti} / \mathrm{SiO}_{2} \mathrm{disks}$. Values are mean metabolic activity \pm standard error of two experiments containing 2-3 repeats $(n=5)$, measured by CTB staining. (c) Eradication of mature $S$. mutans biofilms on $\mathrm{Ti} / \mathrm{SiO}_{2}$ disks. Values are mean metabolic activity \pm standard error of two experiments containing 2-3 repeats $(n=5)$, measured by CTB staining. The asterisks indicate a statistically significant difference in metabolic activity $(* * p<0.01 ; * * * * p<0.0001)$.

setup and $\mathrm{Ti} / \mathrm{SiO}_{2}$ implant experiments) were additionally validated by CFU counting.

To demonstrate that our material was also suitable for treatment of established infections, $S$. mutans biofilms were allowed to grow on the release side of the $\mathrm{Ti} / \mathrm{SiO}_{2}$ disks for $2 \mathrm{~d}$ in the absence of chlorhexidine. Administration of $0.25 \mathrm{mM}$ chlorhexidine to the feed compartment after biofilm maturation significantly reduced the metabolic activity ( $\pm 98 \%$ reduction) of $S$. mutans biofilm cells after only $2 \mathrm{~d}$ of chlorhexidine release (Fig. 3c). CFU counting confirmed that the number of retrieved $S$. mutans biofilm cells was significantly reduced from $1.64 \pm 1.11 \times 10^{6}$ to $2.68 \pm 1.24 \times 10^{4}(p=0.0197)$.

Design and characterisation of mesoporous $\mathrm{Ti} / \mathrm{SiO}_{2}$ implants with an internal reservoir

Next, we developed dental implants consisting of the Ti/ $\mathrm{SiO}_{2}$ composite material (Fig. 1). An internal reservoir was incorporated in the design for customised drug solution storage allowing prolonged release of antimicrobial compounds through the mesoporous $\mathrm{Ti} / \mathrm{SiO}_{2}$ to the implant surface and surroundings.

Fig. 5a shows a backscattered electron image of a cross-sectional detail of the $\mathrm{Ti} / \mathrm{SiO}_{2}$ implant near the screw thread crest, indicating an angular macroporous $\mathrm{Ti}$ phase with open surface pores. Indeed, mercury intrusion porosimetry of the implant indicated that the open porosity amounted $23.0 \%$ while the average interconnecting pore window size was $1.4 \mu \mathrm{m}$ after machining and acid etching (Fig. 5c). A representative image of an associated elemental (Ti and Si overlay) mapping can be found in Fig. 5b. This confirmed the uniform distribution of a $\mathrm{SiO}_{2}$ phase throughout the whole $\mathrm{Ti}$ pore structure in a consistent way. According to nitrogen adsorption measurements, the final $\mathrm{Ti} / \mathrm{SiO}_{2}$ composite dental implant exhibited a pore size distribution ranging from 4 to $10 \mathrm{~nm}$ with an average mesopore diameter of $5.8 \mathrm{~nm}$ and a specific surface area of $180.7 \mathrm{~m}^{2} / \mathrm{g} \mathrm{SiO}_{2}$ (Fig. 5d). 
CONTROL

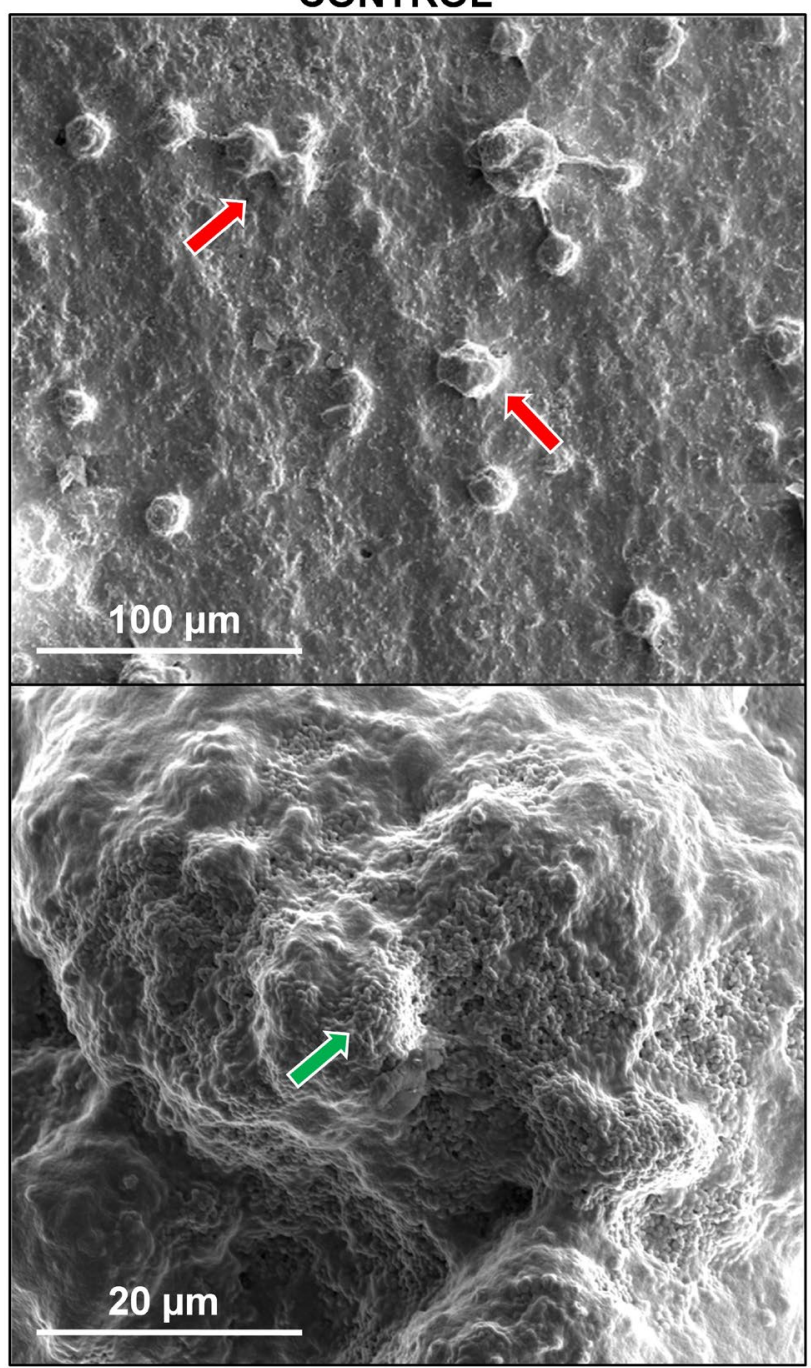

\section{CHLORHEXIDINE}

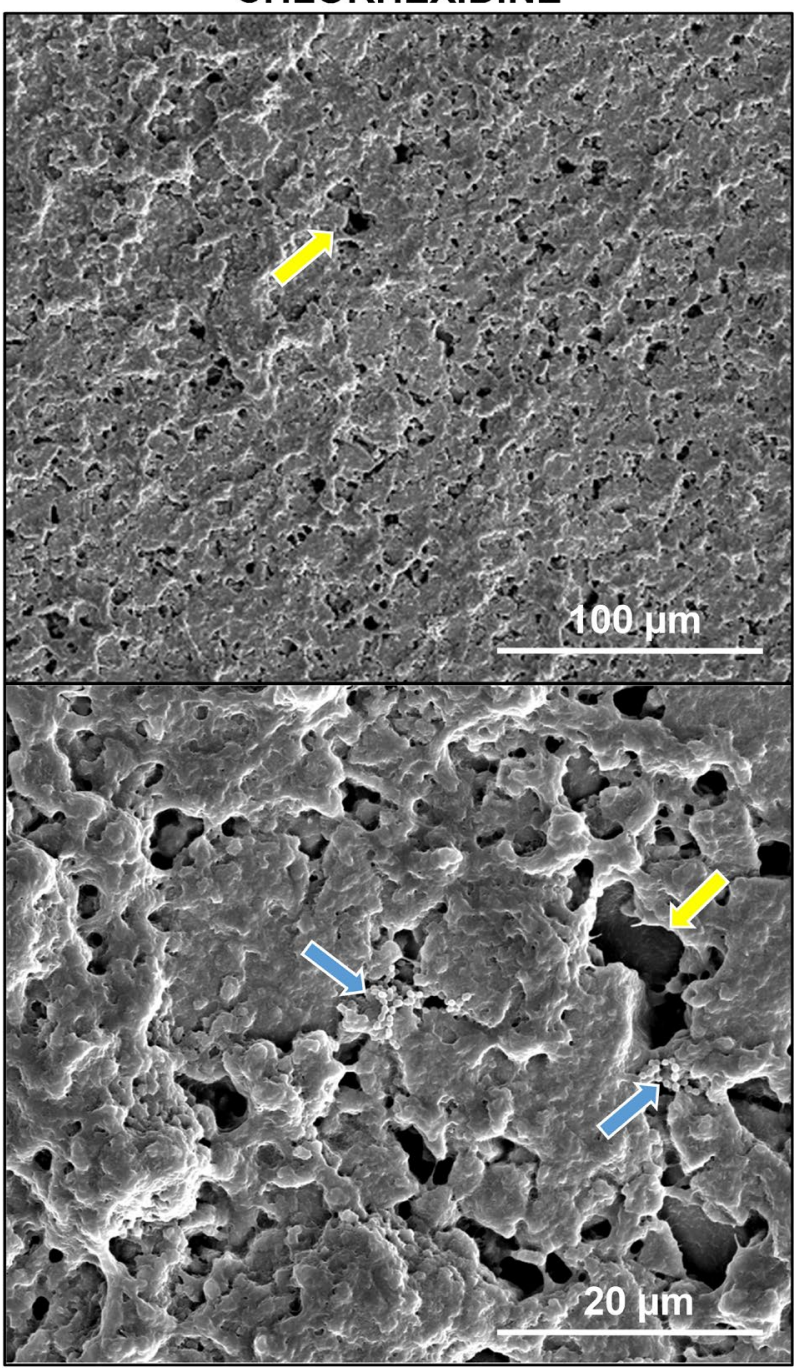

Fig. 4. SEM image of $S$. mutans growth on control (left) and chlorhexidine-releasing (right) $\mathrm{Ti} / \mathrm{SiO}_{2}$ disks. $S$. mutans was allowed to grow on top of $\mathrm{Ti} / \mathrm{SiO}_{2}$ disks following a $10 \mathrm{~d}$ release experiment in which control or $1 \mathrm{mM}$ chlorhexidine solution were applied in the feed compartment of the in vitro test tool (biofilm prevention). The SEM was operated at standard high-vacuum settings at $5 \mathrm{~mm}$ working distance. To avoid beam damage of the biofilm on control disks, low-energy imaging using beam deceleration was performed by applying a stage (and sample) bias field, resulting in an effective landing energy of $5 \mathrm{keV}$. For chlorhexidine-releasing disks, a $10 \mathrm{keV}$ accelerating voltage was used. The formation of mushroom-shaped micro-colonies (red arrows) consisting of S. mutans cells (green arrow) is visible on control disks. On the contrary, the open surface porosity is exposed in chlorhexidinereleasing disks (yellow arrows) which contain only few attached S. mutans cells (blue arrows).

Chlorhexidine is released in a controlled way through $\mathrm{Ti} / \mathrm{SiO}_{2}$ composite implants and inhibits $S$. mutans biofilm formation

Using the dental implants characterised above, we confirmed chlorhexidine release through the $\mathrm{Ti} / \mathrm{SiO}_{2}$ composite material from the internal reservoir $(5 \mathrm{mM}$ chlorhexidine feed solution, approximately $0.3 \%$ ) towards the release side (Fig. 6a). Also in this setup, a steady release of chlorhexidine could be measured from day 3 after the start of the experiment (illustrated by the nearly linear cumulative release profile). On average $26 \mathrm{nmol} / \mathrm{cm}^{2}$ chlorhexidine were released from the internal reservoir, resulting in an average concentration of $80 \mu \mathrm{M}$ in the release medium.
The concentration in the release medium depended on the volume of the release medium and on the volume of the feed solution. Since the ratio of feed volume over release volume is 50 times lower in the implant setup, one can expect lower release concentrations in the implant setup using similar feed concentrations. Indeed, using $5 \mathrm{mM}$ chlorhexidine feed solution, the release concentrations were remarkably lower in the implant setup $( \pm 80 \mu \mathrm{M}$ or $0.005 \%$ ) compared to $1 \mathrm{mM}$ feed concentrations in the disk setup (resulting in an average release concentration of $359 \mu \mathrm{M}$ ). The $5 \mathrm{mM}$ feed concentration resulted in release concentrations in the implant set up that were comparable to release concentrations achieved with 0.1-0.25 mM chlorhexidine feed solutions in the disk setup. Significantly 

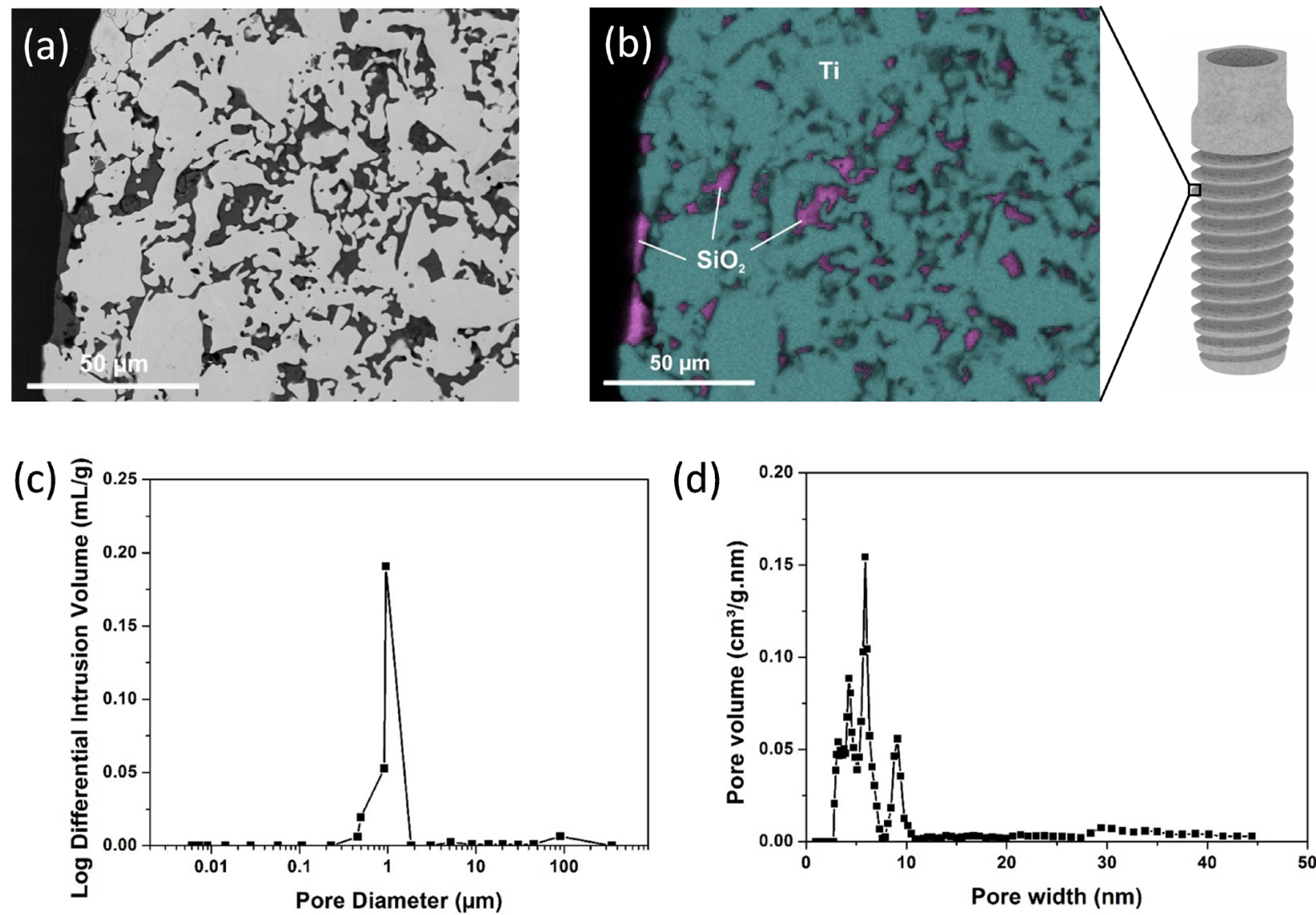

Fig. 5. Combining macro- and mesoporosity in a $\mathrm{Ti} / \mathrm{SiO}_{2}$ composite dental implant. A representative cross-sectional detail at the screw thread crest was analysed by SEM using backscattered electron imaging showing (a) macroporous $\mathrm{Ti}$ (in light grey) and (b) associated elemental mapping confirming the presence of a $\mathrm{SiO}_{2}$ phase (magenta) inside the $\mathrm{Ti}$ (blue) macropores in a consistent way. (c) Prior to $\mathrm{SiO}_{2}$ infiltration, the macroporous Ti implant has a pore window size distribution around $1.4 \mu \mathrm{m}$ as measured by mercury intrusion porosimetry, while the $(\mathbf{d}) \mathrm{Ti} / \mathrm{SiO}{ }_{2}$ composite dental implant presents a mesoporous structure with an average pore size of $5.8 \mathrm{~nm}$ as determined by nitrogen adsorption measurements.

(a)

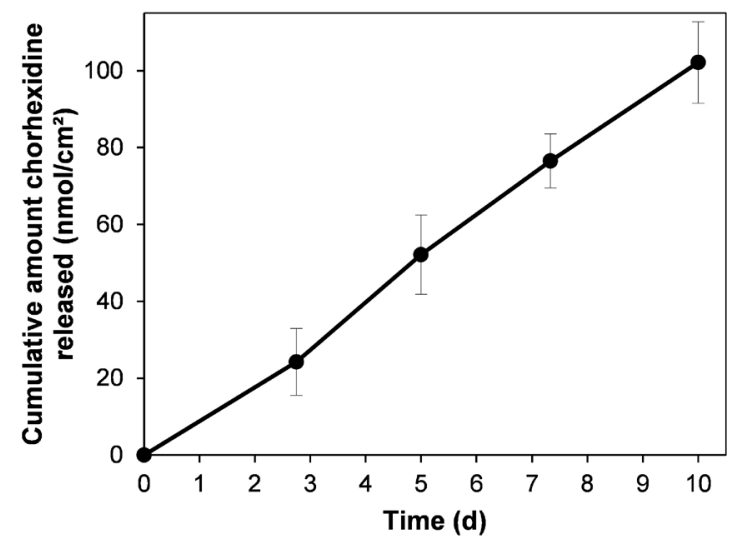

(b)

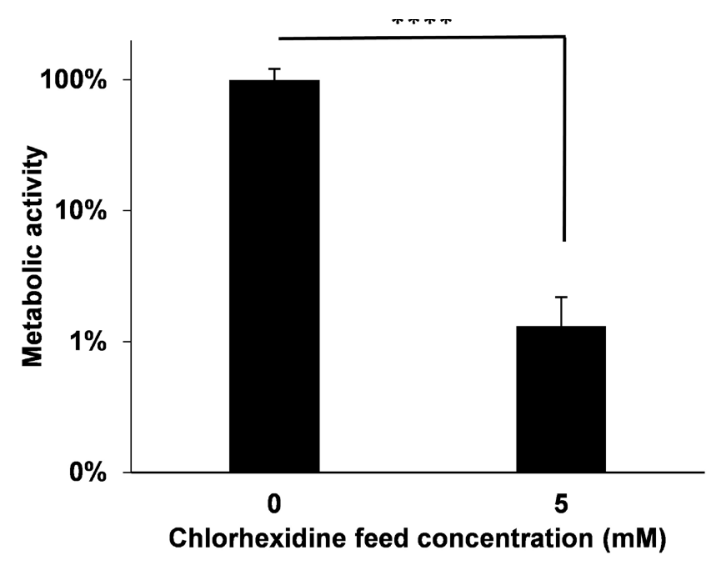

Fig. 6. Release of chlorhexidine through $\mathrm{Ti} / \mathrm{SiO}_{2}$ implant and associated effect on $\mathrm{S}$. mutans metabolic activity. (a) Cumulative amount of chlorhexidine released through $\mathrm{Ti} / \mathrm{SiO}_{2}$ implants in the in vitro test tool for $5 \mathrm{mM}$ chlorhexidine feed concentration (measured by UV spectrophotometry at $255 \mathrm{~nm}$ ). Values are mean \pm standard error $(n=3)$. (b) Metabolic activity of $S$. mutans biofilms grown for $72 \mathrm{~h}$ on $\mathrm{Ti} / \mathrm{SiO}_{2}$ implants on the implant surface after $10 \mathrm{~d}$ of chlorhexidine release. Values are mean \pm standard error of two experiments containing 3 repeats $(n=6)$, measured by CTB staining. The asterisks indicate a statistically significant difference in metabolic activity $(* * * * p<0.0001)$. 


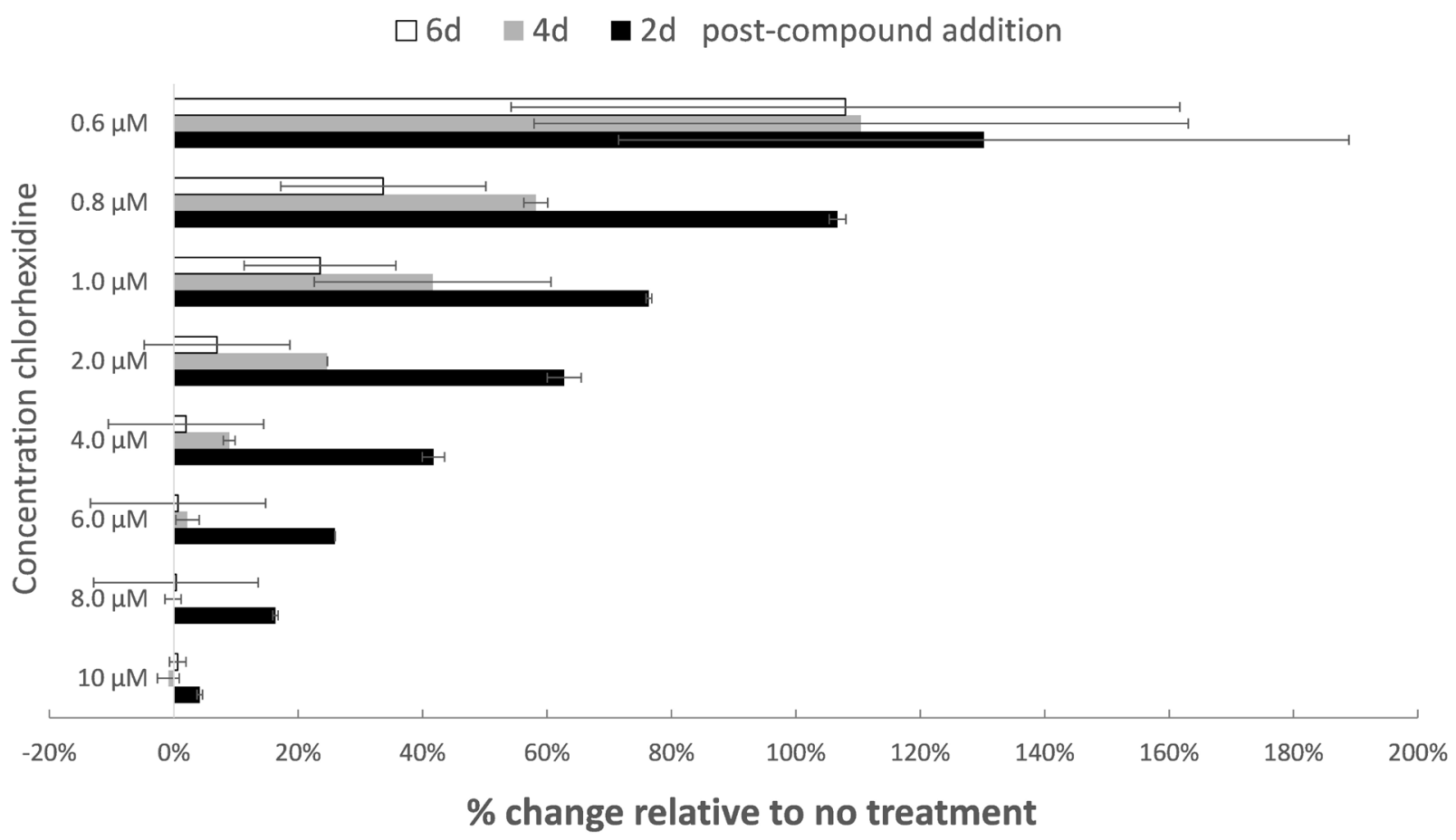

Fig. 7. Effect of chlorhexidine on the viability and growth potential of osteoblast-like cells. MG63 cells were grown in 24-well plates in $\alpha$ MEM. At day 2 post-seeding, the cells were incubated with or without chlorhexidine by adding the compound or mock treatment to the culture medium. MG63 proliferation profiles were evaluated using Presto Blue measurements relative to the no treatment control on the same day, with measurements after 2, 4 and $6 \mathrm{~d}$ of compound addition. This figure represents two independent biological experiments consisting of two technical repeats each.

reduced metabolic activity ( $\pm 99 \%$ reduction) of $S$. mutans cells growth on chlorhexidine-releasing implants $(5 \mathrm{mM}$ feed concentration) was observed compared to $S$. mutans biofilm cells grown on control implants (Fig. 6b).

We confirmed these observations by CFU counting of $S$. mutans biofilm cells recuperated from the implants. Biofilms grown on control $\mathrm{Ti} / \mathrm{SiO}_{2}$ implants consisted of on average $2.01 \pm 0.55 \times 10^{7} \mathrm{CFU} /$ implant, whereas no CFUs could be retrieved from chlorhexidine-releasing implants. This is, however, an underestimation of the number of recovered CFUs, since $S$. mutans cells could still be detected on the implant surface after sonication (by SEM imaging; data not shown). Nevertheless, it was clear that the residual biofilm was considerably larger on control $\mathrm{Ti} / \mathrm{SiO}$ implants than on chlorhexidine-releasing implants, where only a few remaining cells were observed. This indicated that, although sonication was not sufficient for a complete removal of established biofilms from the implant substrate, CFU counting of recuperated S. mutans cells still allowed for a qualitative comparison between the different samples. Moreover, the minimal dilution that was plated on agar was a 10-fold dilution of the suspension in which the biofilm was dissolved, thereby thresholding our quantification to $10 \mathrm{CFUs}$ /biofilm.

\section{Chlorhexidine affects the growth potential of} osteoblast-like cells in a dose-dependent manner Osteoblasts are key players in the processes of establishment and maintenance of implant osseointegration. Therefore, in view of the application of chlorhexidine in preventing/ curing peri-implantitis, we examined the cytotoxic effects of chlorhexidine on MG63 osteoblast-type cells (Fig. 7). At day 2 post-seeding, cells were incubated with chlorhexidine by adding different concentrations of the compounds to the culture medium. To determine the toxicity of chlorhexidine as a function of the various applied concentrations, cell proliferation was determined after 2, 4 and $6 \mathrm{~d}$ of incubation and normalised to the no treatment control on the same day. Upon treatment with chlorhexidine up to $1.0 \mu \mathrm{M}$, human osteoblast-like cells survived and cell proliferation was permitted, however cytotoxic behaviour (less than $70 \%$ proliferation compared to the no treatment control) of chlorhexidine towards the osteoblasts was apparent for doses above $0.6 \mu \mathrm{M}$ when cells were treated for more than two days.

\section{Discussion}

Implant related infections are a serious complication in prosthetic surgery. Recently, implants with rough surfaces or porous surface coatings are applied for improved osseointegration. However, some studies report that the increased surface roughness renders the implant more susceptible to microbial colonisation and subsequent biofilm formation (Belibasakis et al., 2015; Braem et al., 2014; Subramani et al., 2009). Therefore, the development of implant materials or surfaces that combine both the ability to improve osseointegration and at the same time reduce the infection risk are of interest. The unique implant 
design proposed in this study exposes a rough implant surface, while overcoming the reported associated problem of increased bacterial growth by controlled release of chlorhexidine, which is loaded in the internal reservoir, through the $\mathrm{Ti} / \mathrm{SiO}_{2}$ composite material.

The capacity of this $\mathrm{Ti} / \mathrm{SiO}_{2}$ composite material to transfer the antimicrobial compound chlorhexidine from a feed compartment to a release compartment was assessed in our in vitro test tool consisting of $\mathrm{Ti} / \mathrm{SiO}_{2}$ disks. It was demonstrated that chlorhexidine diffuses steadily through the mesoporous material over at least $10 \mathrm{~d}$ (Fig. 3a) and that therapeutically relevant concentrations can be released up to $40 \mathrm{~d}$. The kinetic diameter of chlorhexidine having a molecular size of $c a .3 .4 \times 0.7 \times 0.6 \mathrm{~nm}$ is within the same range as the pore size (circa $6 \mathrm{~nm}$ ) of the $\mathrm{SiO}_{2}$ phase of the $\mathrm{Ti} / \mathrm{SiO}_{2}$ composite, therefore, the diffusion is governed by a configurational regime as indicated by the apparent diffusivity in the order of $10^{-11} \mathrm{~m}^{2} / \mathrm{s}$ (Verraedt et al., 2010).

We assessed the activity of the released chlorhexidine against $S$. mutans biofilms, both in a preventive (to prevent biofilm formation) and curative setup (to eradicate existing biofilms). The choice of the bacterium $S$. mutans in this study should be interpreted as most relevant in the preventive set-up - prevention of the biofilm composition maturation towards anaerobic species by eradicating the preceding aerobic ones. The released chlorhexidine was capable of almost completely preventing $S$. mutans biofilm formation on the release side of the $\mathrm{Ti} / \mathrm{SiO}_{2}$ disk (Fig. 3b and Fig. 4). In a similar setup, the anti-biofilm effect of toremifene was previously reported against the fungal pathogen Candida albicans (Braem et al., 2015), demonstrating the broad flexibility of this system to act against different biofilm-forming pathogens and releasing diverse compounds. Moreover, by adjusting the concentration of the feed solution, the amount of released compound can be fine-tuned (Fig. 3a). These features are unique for drug-releasing implant materials, since most implant materials suitable for controlled release of a medical drug are preloaded, releasing only one compound until depletion. Moreover, using these materials, the drug is mainly leached during an initial burst release followed by release at sub-therapeutic concentrations, risking resistance development (Goodman et al., 2013; Lyndon et al., 2014). It should be noted that compounds administered into the periodontal pocket are rapidly washed out in vivo (Goodson, 2003). To mimic this situation, we refreshed the release medium every $2 \mathrm{~d}$ to maintain sink conditions. GCF flow rates are highly dependent on the disease state with higher values with increasing infection status (from $5 \mu \mathrm{L} / \mathrm{h}$ up to $137 \mu \mathrm{L} / \mathrm{h}$ ). Fine-tuning of a continuous flow system for this set-up, thereby discriminating between healthy persons and patients with intermediate or advanced periodontal disease would be most interesting.

Using the $\mathrm{Ti} / \mathrm{SiO}_{2}$ disks, we additionally demonstrated activity of released chlorhexidine against mature $S$. mutans biofilms (in a curative setup) (Fig. 3c), both using metabolic staining and CFU counting. Consequently, treatment of infections occurring in later stages of implant therapy is possible as well, by adding the desired agent in the reservoir, which is easily accessible. Moreover, this confirms that the observed effect is not merely due to a surface effect but that the chlorhexidine actively kills $S$. mutans biofilm cells.

Controlled release of compounds from the internal cavity through the implant material is a highly novel approach. However, various existing dental implants consist of an internal cavity, not for drug release but to enable dental surgery. Such cavity allows engaging specific surgery tools or screw-retained fixtures, like healing screws, abutments or crowns. The concept of combining hollow tubular metallic support structures including orifices of 0.2 up to $1.0 \mathrm{~mm}$ with various drug-releasing carrier materials inside the internal cavity has been previously suggested, among others for percutaneous orthopaedic implants (Clark et al., 2008; Gimeno et al., 2015; Park et al., 2014; Perez et al., 2011; Santos et al., 2014). The novelty of the current design lies in the fact that the mesoporous controlled release $\left(\mathrm{SiO}_{2}\right)$ matrix is incorporated in the (Ti) implant walls (Fig. 1 and Fig. 5a, b), rendering the functional implant itself a true drug delivery vehicle. Indeed, current results indicate that the previously described processing route for the $\mathrm{Ti} / \mathrm{SiO}_{2}$ composite material (Braem et al., 2015) was successfully transferred to the proposed dental implant design as indicated by the comparable macro- and mesoporous characteristics (Fig. 5c, d). The controlled release of the molecules applied in the reservoir is governed by their diffusion through the mesoporous $\mathrm{SiO}_{2}$ phase. It is well known that by controlling the pore morphology and/ or chemistry of $\mathrm{SiO}_{2}$ materials the diffusion (and hence the release kinetics) of therapeutic molecules can be fine-tuned (Aerts et al., 2010; Bhaskara et al., 2014; Bhattacharyya et al., 2014; Santamaria et al., 2014; Verraedt et al., 2010). We hypothesise that incorporating a $\mathrm{SiO}_{2}$ material with adequate pore characteristics will allow to adapt the system for any specific molecule. Moreover, there is no risk for bacteria to enter the implant, since the mesopores in the $\mathrm{Ti} / \mathrm{SiO}_{2}$ are only a few $\mathrm{nm}$ in diameter, which is too small for bacteria to enter.

Up to now, the clinical implementation of localised drug delivery around dental implants has been limited to the application of drug eluting coatings on the implant surface or the injection of bioresorbable drug carriers into the periimplant pocket (Santos et al., 2014). While coatings are fragile and can easily get damaged upon implant insertion (Hickok and Shapiro, 2012), drug carrier materials in the peri-implant gingival pockets are subject to erosion due to masticatory action. With this drug-releasing functional dental implant, a more reliable and more homogeneously distributed antimicrobial release over the entire implant surface area is envisaged and over an extended time period. By applying a $5 \mathrm{mM}$ (approximately $0.3 \%$ ) chlorhexidine feed solution in the implant reservoir, we obtained a steady release resulting in on average $80 \mu \mathrm{M}$ chlorhexidine (approximately $0.005 \%$ ) in the release medium over at least $10 \mathrm{~d}$ (Fig. 6a). However, based on the results from the extended $\mathrm{Ti} / \mathrm{SiO}_{2}$ disk experiment, we also can expect a much longer release of active chlorhexidine concentrations from the $\mathrm{Ti} / \mathrm{SiO}_{2}$ implant.

Many studies have reported benefits associated with chlorhexidine treatment as preventive therapy against development of peri-implant disease as adjunct to mechanical debridement and basic oral hygiene (Pedrazzi 
et al., 2014). Notwithstanding its efficacy, evidence is increasing that chlorhexidine may have deleterious effects on various cell types in vitro (Pucher and Daniel, 1992; Cline and Layman, 1992; Mariotti and Rumpf, 1999; Giannelli et al. 2008). Given that osteoblasts represent the main cell type involved in bone tissue regeneration and their function is pivotal for the clinical resolution of periodontal and peri-implant defects (Shibli et al., 2006), the use of chlorhexidine in periodontal and peri-implant complications should not affect the osteoblasts and trouble tissue homeostasis and healing. The obtained in vitro data confirm the cytotoxicity of chlorhexidine directly applied onto osteoblasts when used at concentrations exceeding $1 \mu \mathrm{M}$ (approximately $0.00006 \%$ ). The direct application of chlorhexidine at higher concentrations during regenerative therapy for the treatment of peri-implantitis could thus have serious toxic effects on alveolar osteoblasts. However, the use of chlorhexidine mouthwashes $(0.12 \%)$ after implant installation is almost routine practice to maintain periimplant health. Obviously, the contact is only for a few seconds or minutes via mucous membrane in this case. Interestingly, patients irrigating with diluted chlorhexidine $(0.06 \%$, which is still far above the concentrations released in our setup) using a powered oral irrigator with a special subgingival irrigating tip showed reduced infection symptoms compared to patients rinsing with $0.12 \%$ chlorhexidine gluconate once daily and is recommended as a valuable adjunct to oral health in patients with dental implants (Felo et al., 1997). These results suggest that tackling the zone most proximal to the implant results in superior health outcome. This is precisely what is aimed for with the dental implant presented in this study, as it is capable of releasing chlorhexidine directly at the implant surface. Moreover, the observed chlorhexidine release concentrations of $0.005 \%$ were sufficient to prevent $S$. mutans biofilm growth on the implant surface, suggesting that very low doses of an antibacterial compound are adequate when administered locally during a limited time. Due to the complex setup of the experiments it was not feasible to repeat the experiments using a lower range of chlorhexidine to determine the critical release concentration. However, recalling the reported MIC/BIC values of 1-3 $\mu \mathrm{M}$, we estimate that a few $\mu \mathrm{M}$ would suffice, which is comparable to the reported cytotoxicity limit. Hence, in follow-up experiments, less cytotoxic compounds should additionally be evaluated for compatibility with our dental implant release system, for which chlorhexidine can serve as benchmark.

Indeed, our design allows for the insertion of other antibacterial compounds in the internal reservoir instead of chlorhexidine, such as antibiotics, thereby installing a very local antibiotics regimen for a prolonged time at the place of potential infections. Such local antibiotic dosing regimen, avoiding their systemic use, can be very attractive as the latter is associated with antibiotic resistance development. Moreover, the design of the Ti/ $\mathrm{SiO}_{2}$ composite dental implant presented in this manuscript enables a personalised treatment. Different antimicrobial compounds or antibiotics can be applied in the internal reservoir depending on the patient's need. Moreover, it is hypothesised that consecutive release of different antimicrobial compounds or higher doses of the initial compound is possible by refilling the reservoir. This might be of interest in cases where the applied drug/dose appears to be ineffective. Additionally, simultaneous release of multiple compounds is envisaged as well, since combination therapy offers several advantages compared to single compound treatment of both bacterial and fungal biofilm infections (Blackledge et al., 2013; De Cremer et al., 2015). Once clinical and radiographic data indicate successful implant osseointegration, there is obviously no need for continued prophylaxis (preventive setup). If signs of peri-implantitis would emerge on osseointegrated implants at a later stage, the treatment by reversible, controlled release and repeatable drug administration can be started and stopped at any time (curative setup).

In conclusion, we provide a proof of concept of the sustained release of an antimicrobial compound from an internal reservoir through the $\mathrm{Ti} / \mathrm{SiO}_{2}$ material to the implant outer surface and surroundings. Release of this antimicrobial compound prevents and eradicates microbial biofilm formation on the rough implant surface, decreasing the risk for development of peri-implant diseases. Responding to the current need for more personalised treatments, our flexible implant design allows for the use of a broad range of antimicrobial compounds (alone or in combination) and fine-tuning of the concentration at which these are released will depend on the patient's need. This study focuses on dental implant applications, but the concept could, in principle, be translated to any percutaneous implant that can incorporate an internal reservoir, such as percutaneous fixation devices, as long as this does not compromise the mechanical strength of such load-bearing devices.

\section{Acknowledgements}

The research leading to these results has received funding from the Industrial Research Fund of KU Leuven by the knowledge platform IOF/KP/11/007 and the Flemish government by the Hercules Foundation (projectZW09-09). K.T. acknowledges the receipt of a mandate from the Industrial Research Fund (IOFm/05/022, KU Leuven) and K.D.C. acknowledges the receipt of a mandate from FWOVlaanderen (12F0516N). The authors would like to thank Dr Nicolas Delattin and Els Meert (Centre of Microbial and Plant Genetics) for assistance with the preliminary setup of chlorhexidine release experiments and Annelies Peeters (Centre of Microbial and Plant Genetics) for overall technical support during the release experiments.

\section{References}

Aerts CA, Verraedt E, Depla A, Follens L, Froyen L, Van Humbeeck J, Augustijns P, Van den Mooter G, Mellaerts R, Martens JA (2010) Potential of amorphous microporous silica for ibuprofen controlled release. Int $\mathrm{J}$ Pharm 397: 84-91. 
Belibasakis GN, Charalampakis G, Bostanci N, Stadlinger B (2015) Peri-implant infections of oral biofilm etiology. Adv Exp Med Biol 830: 69-84.

Bencharit S, Byrd WC, Altarawneh S, Hosseini B, Leong A, Reside G, Morelli T, Offenbacher S (2014) Development and applications of porous tantalum trabecular metal-enhanced titanium dental implants. Clin Implant Dent Relat Res 16: 817-826.

Bhaskara Rao B V, Mukherji R, Shitre G, Alam F, Prabhune AA, Kale SN (2014) Controlled release of antimicrobial Cephalexin drug from silica microparticles. Mater Sci Eng C Mater Biol Appl 34: 9-14.

Bhattacharyya S, Agrawal A, Knabe C, Ducheyne P (2014) Sol-gel silica controlled release thin films for the inhibition of methicillin-resistant Staphylococcus aureus. Biomaterials 35: 509-517.

Blackledge MS, Worthington RJ, Melander C (2013) Biologically inspired strategies for combating bacterial biofilms. Curr Opin Pharmacol 13: 699-706.

Braem A, Van Mellaert L, Mattheys T, Hofmans D, De Waelheyns E, Geris L, Anné J, Schrooten J, Vleugels J (2014) Staphylococcal biofilm growth on smooth and porous titanium coatings for biomedical applications. J Biomed Mater Res A 102: 215-224.

Braem A, De Cremer K, Delattin N, De Brucker K, Neirinck B, Vandamme K, Martens JA, Michiels J, Vleugels J, Cammue BPA, Thevissen K (2015) Novel anti-infective implant substrates: controlled release of antibiofilm compounds from mesoporous silica-containing macroporous titanium. Colloids Surf B Biointerfaces 126: 481-488.

Busscher HJ, Rinastiti M, Siswomihardjo W, van der Mei HC (2010) Biofilm formation on dental restorative and implant materials. J Dent Res 89: 657-665.

Campoccia D, Montanaro L, Arciola CR (2013) A review of the biomaterials technologies for infectionresistant surfaces. Biomaterials 34: 8533-8554.

Clark PA, Moioli EK, Sumner DR, Mao JJ (2008) Porous implants as drug delivery vehicles to augment host tissue integration. FASEB J 22: 1684-1693.

Clarke JK (1924) On the bacterial factor in the aetiology of dental caries. Br J Exp Pathol 5: 141-147.

Cline NV, Layman DL (1992) The effects of chlorhexidine on the attachment and growth of cultured human periodontal cells. J Periodontol 63: 598-602.

Dalwai F, Spratt DA, Pratten J (2006) Modeling shifts in microbial populations associated with health or disease. Appl Environ Microbiol 72: 3678-3684.

De Cremer K, Staes I, Delattin N, Cammue BP, Thevissen K, De Brucker K (2015) Combinatorial drug approaches to tackle Candida albicans biofilms. Expert Rev Anti Infect Ther 13: 973-984.

de Freitas MM, da Silva CHP, Groisman M, Vidigal GM (2011) Comparative analysis of microorganism species succession on three implant surfaces with different roughness: an in vivo study. Implant Dent 20: e14-23.

do Prado RF, de Vasconcellos LGO, de Vasconcellos LMR, Cairo CAA, Leite Dde O, dos Santos A, Jorge AO, Romeiro Rde L, Balducci I, Carvalho YR (2013) In vivo osteogenesis and in vitro Streptococcus mutans adherence: porous-surfaced cylindrical implants vs. rough-surfaced threaded implants. Int J Oral Maxillofac Implants 28: 1630-1638.

Donelli G, Vuotto C (2014) Biofilm-based infections in long-term care facilities. Future Microbiol 9: 175-188.

Esposito M, Coulthard P, Thomsen P, Worthington H V (2005) Interventions for replacing missing teeth: different types of dental implants. Cochrane database Syst Rev: CD003815.

Felo A, Shibly O, Ciancio SG, Lauciello FR, Ho A (1997) Effects of subgingival chlorhexidine irrigation on peri-implant maintenance. Am J Dent 10: 107-110.

Giannelli M, Chellini F, Margheri M, Tonelli P, Tani A (2008) Effect of chlorhexidine digluconate on different cell types: a molecular and ultrastructural investigation. Toxicol In Vitro 22: 308-317.

Gimeno M, Pinczowski P, Pérez M, Giorello A, Martínez MÁ, Santamaría J, Arruebo M, Luján L (2015) A controlled antibiotic release system to prevent orthopedicimplant associated infections: An in vitro study. Eur J Pharm Biopharm 96: 264-271.

Goodman SB, Yao Z, Keeney M, Yang F (2013) The future of biologic coatings for orthopaedic implants. Biomaterials 34: 3174-3183.

Goodson JM (2003) Gingival crevice fluid flow. Periodontol 2000 31: 43-54.

Hasan J, Crawford RJ, Ivanova EP (2013) Antibacterial surfaces: the quest for a new generation of biomaterials. Trends Biotechnol 31: 295-304.

Hickok NJ, Shapiro IM (2012) Immobilized antibiotics to prevent orthopaedic implant infections. Adv Drug Deliv Rev 64: 1165-1176.

Junka AF, Szymczyk P, Smutnicka D, Kos M, Smolina I, Bartoszewicz M, Chlebus E, Turniak M, Sedghizadeh PP (2015) Microbial biofilms are able to destroy hydroxyapatite in the absence of host immunity in vitro. $\mathrm{J}$ Oral Maxillofac Surg 73: 451-64.

Lyndon JA, Boyd BJ, Birbilis N (2014) Metallic implant drug/device combinations for controlled drug release in orthopaedic applications. J Control Release 179: 63-75.

Marcantonio C, Nicoli LG, Junior EM, ZandimBarcelos DL (2015) Prevalence and Possible Risk Factors of Peri-implantitis: A Concept Review. J Contemp Dent Pract 16: 750-7.

Mariotti AJ, Rumpf DA (1999) chlorhexidine-induced changes to human gingival fibroblast collagen and noncollagen protein production. J Periodontol 70: 1443-1448.

Nicu EA, Van Assche N, Coucke W, Teughels W, Quirynen M (2012) RCT comparing implants with turned and anodically oxidized surfaces: a pilot study, a 3-year follow-up. J Clin Periodontol 39: 1183-1190.

Park YS, Cho JY, Lee SJ, Hwang CI (2014) Modified titanium implant as a gateway to the human body: the implant mediated drug delivery system. Biomed Res Int 2014: 801358.

Pedrazzi V, Escobar EC, Cortelli JR, Haas AN, Andrade AK, Pannuti CM, Almeida ER, Costa FO, Cortelli SC, Rode Sde M (2014) Antimicrobial mouthrinse use as an adjunct method in peri-implant biofilm control. Braz Oral Res 28. 
Perez LM, Lalueza P, Monzon M, Puertolas JA, Arruebo M, Santamaría J (2011) Hollow porous implants filled with mesoporous silica particles as a two-stage antibiotic-eluting device. Int J Pharm 409: 1-8.

Pucher JJ, Daniel JC (1992) The effects of chlorhexidine digluconate on human fibroblasts in vitro. J Periodontol 63: 526-532.

Ribeiro M, Monteiro FJ, Ferraz MP (2012) Infection of orthopedic implants with emphasis on bacterial adhesion process and techniques used in studying bacterial-material interactions. Biomatter 2: 176-194.

Santamaría E, Maestro A, Porras M, Gutiérrez JM, González C (2014) Controlled release of ibuprofen by meso-macroporous silica. J Solid State Chem 210: 242250.

Santos A, Sinn Aw M, Bariana M, Kumeria T, Wang Y, Losic D (2014) Drug-releasing implants: current progress, challenges and perspectives. J Mater Chem B 2: 6157.

Shibli JA, Martins MC, Ribeiro FS, Garcia VG, Nociti FH, Marcantonio E (2006) Lethal photosensitization and guided bone regeneration in treatment of peri-implantitis: an experimental study in dogs. Clin Oral Implants Res 17: 273-281.

Subramani K, Jung RE, Molenberg A, Hammerle CHF (2009) Biofilm on dental implants: a review of the literature. Int J Oral Maxillofac Implants 24: 616-626.

Taniguchi Y, Kakura K, Yamamoto K, Kido H, Yamazaki J (2015) Accelerated osteogenic differentiation and bone formation on zirconia with surface grooves created with fiber laser irradiation. Clin Implant Dent Relat Res 18: 883-894.

Varoni E, Tarce M, Lodi G, Carrassi A (2012) chlorhexidine $(\mathrm{CHX})$ in dentistry: state of the art. Minerva Stomatol 61: 399-419.

Verraedt E, Pendela M, Adams E, Hoogmartens J, Martens JA (2010) Controlled release of chlorhexidine from amorphous microporous silica. J Control Release 142: 47-52.

Yuan K, Chan YJ, Kung KC, Lee TM (2014) Comparison of osseointegration on various implant surfaces after bacterial contamination and cleaning: a rabbit study. Int J Oral Maxillofac Implants 29: 32-40.

\section{Discussion with Reviewer}

Llinos Harris: How effective is the system on the other bacteria that are commonly present in the mouth? Is it possible that the eradication of $S$. mutans could lead to other species flourishing and causing infections?

Authors: Chlorhexidine is known to be a broad-spectrum biocide effective against Gram-positive bacteria, Gramnegative bacteria and fungi. To show the effectiveness of our system, we have performed additional experiments and demonstrated biofilm-preventing activity of chlorhexidine released through $\mathrm{Ti} / \mathrm{SiO}_{2}$ disks against Candida albicans biofilms, a yeast species known to be involved in dental infections (Fig. 8).

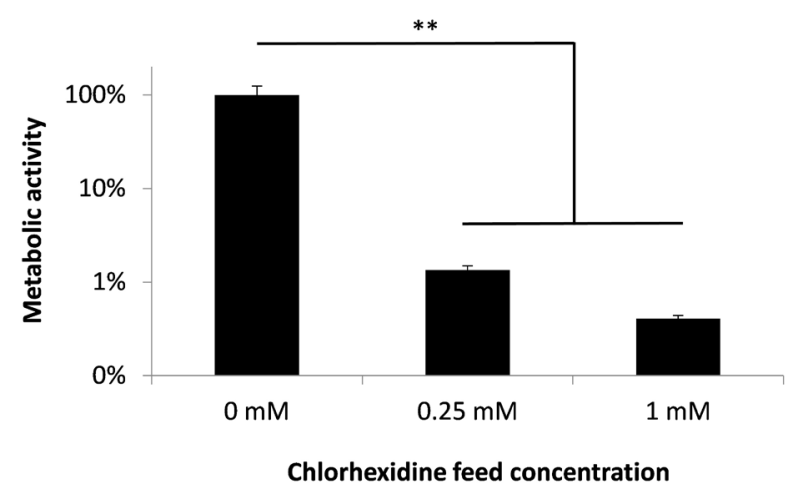

Fig. 8. Prevention of $C$. albicans biofilm formation on chlorhexidine-releasing $\mathrm{Ti} / \mathrm{SiO}_{2}$ disks. Different concentrations of chlorhexidine were applied to the feed reservoir in the in vitro test tool. Values are mean metabolic activity of $72 \mathrm{~h}$ old $C$. albicans biofilms \pm standard error $(n=3)$, measured by CTB staining. The asterisks indicate a statistically significant difference in metabolic activity $(* * p<0.01)$.

Llinos Harris: Do the authors know what would happen to the osteoblasts if exposed to the initial burst release observed in the first $3 \mathrm{~d}$ ?

Authors: We have assessed release kinetics of chlorhexidine (Fig. 3a) and we described a steady release of chlorhexidine from day 3 without initial burst (resulting in mean chlorhexidine release of $9 \mathrm{nmol} / \mathrm{cm}^{2}$ ) for the lowest feed concentration $(0.1 \mu \mathrm{M}$ chlorhexidine $)$. In our set-up, this corresponds to $33 \mu \mathrm{M}$ chlorhexidine - a dose with in vitro toxicity to osteoblasts. However, it should be noted that compounds administered into the periodontal pocket are rapidly washed out in vivo (Goodson, 2003). Therefore we anticipate that in vivo chlorhexidine release is gradually cleared by the surrounding tissue, resulting in lower net chlorhexidine concentrations. Hence, it is very difficult to predict in vivo toxicity associated with chlorhexidine release from our device based on the current in vitro data. As a consequence, the data from this study have to be seen in a broader context, namely as a proof of concept, which can be extended to the use of any bioactive, potentially less toxic, compound.

Britt Wildermann: Can you make any assumption regarding the effect of cells growing on the implant and the release kinetics. Due to the fact that the implant should be integrated, the pores might be filled/covered by cells. Would this then stop the release and hinder the envisioned use to cure a late infection?

Authors: At present, we have no data on the effect of osteoblast growth on the material and potential clogging of the mesopores, thereby hindering release of the antimicrobial compound. In vivo follow-up studies are necessary to provide data in this regard. Nevertheless, in a preventive set-up, no cells are present immediately after implant installation when infections are most common to occur. When compounds should be administered for 
curative reasons, the infection is often associated with bone loss which will (re-)enable release of compounds.

Britt Wildermann: Information regarding the mechanical properties would be interesting. Do these implants fulfil the requirements of dental implants?

Authors: This important issue will need to be explored in order to take the current proof of concept further to a potential clinical implementation. The presence of micropores in the titanium implant will inherently reduce its mechanical strength, while fatigue cracks can initiate at the open surface pores reducing its endurance limit. However, it is not well understood how the presence of a second phase $\left(\mathrm{SiO}_{2}\right)$ inside the micropores will influence the mechanical behaviour of the composite material. Therefore, future work will focus on the characterisation of the mechanical properties of the designed implants.

Reto Luginbuehl: Do you have data on blocking of the pores by adsorbed proteins or calcification?

Authors: The pores of the silica correspond to the void volumes generated by crosslinking of spherical nanoparticles. The $3 \mathrm{D}$ porous network facilitates molecular transport and minimises the risk of pore blockage, especially for relatively smaller molecules like chlorhexidine. Currently, there is no data available on how the adsorption of macromolecules/proteins (from the implant environment) or bone formation on the implants outer surface will influence the controlled release of the bioactive target molecules (such as the chlorhexidine used in this study). The controlled release is a diffusion process driven by a concentration difference of the target molecule between the internal reservoir and the implant surroundings. We anticipate that the presence of proteins or bone on the implant surface will slow down the dissipation of the target molecule and lead to a local build-up. As such, the driving force for the diffusion through the implant will be less. However, when infections occur, this is usually accompanied by bone loss, so that the concentration of the target molecule at the implant surface will drop again, in turn increasing the release from the implant.

T. Fintan Moriarty: Does the internal cavity/reservoir refill with physiological fluids after antimicrobials have released? Presumably, the release of antibiotic will not result in a vacuum within the implant and if the reservoir can be refilled with fluid entering between the cover screw and implant, could the reservoir also become colonised by oral microflora?

Authors: Indeed, we hypothesise that release of the antimicrobial agent from the reservoir will not result in a vacuum, but will probably allow water molecules (which can migrate through the mesopores) to migrate to the cavity. However, this will not be possible for the microbial cells as these are too big (approx. 0.2-1 $\mu \mathrm{m}$ micro-organism size versus $6 \mathrm{~nm}$ pore size). As indicated in the Materials and Methods section, we used a stainless steel cover screw $(\mathrm{M} 2 \times 2.25)$ for flexible refilling of the internal reservoir. This cover screw is equipped with a conical surface mating the $2^{\circ}$ countersink in the implant abutment in order to provide a sealing surface that effectively prevents leakage of the drug solution to the oral cavity and vice versa. Hence, we do not anticipate that fluid can enter the cavity through the cover screw.

Editor's note: The Scientific Editor responsible for this paper was T. Fintan Moriarty. 